\author{
AMSTERDAM GRADUATE PHILOSOPHY CONFERENCE 2010 \\ Department of Philosophy \\ Institute for Logic, Language and Computation \\ Universiteit van Amsterdam \\ 30 September - 2 October 2010
}

\title{
Pleonastic Possible Worlds
}

\author{
Alex Steinberg \\ Humboldt Universität zu Berlin and University College London
}

\begin{abstract}
The standard semantics for the modal fragment of natural languages can only be correct if there is more than one possible world. The nature and existence of possible worlds is thus of fundamental importance for such a semantics. The paper develops an account of possible worlds in the spirit of the pleonastic account of abstract entities in general, due to Stephen Schiffer (2003: ch. 2). The main motivation is that possible worlds share a telling feature with other abstracta like properties and propositions: our canonical epistemic access to them is via transformations that take us from sentences in which these entities are not mentioned or quantified over to sentences in which they are so-called something-fromnothing transformations. Definitions are proposed that ensure that these transformations turn out to be valid as a matter of conceptual necessity.
\end{abstract}

\section{References}

Schiffer, S. 2003: The Things We Mean. Oxford: Clarendon Press. 